\title{
determinación de la superficie específica por medio de un nomograma
}

\author{
A. VIRELLA \\ Director de la fábrica de cementos blancos eCIBRA= (Portugal)
}

\section{EXPRESIONES DE LA FINURA}

Las industrias que, como la del cemento, manejan sólidos pulverulentos, han tenido una preocupación constante sobre los procedimientos para determinar la finura de los polvos y la forma de expresarla numiticamente.

La expresión más simple, antigua y cotriente es la de un residuo (tanto por ciento de peso de material) que no atraviesa las mallas de un tamiz de abertura conocida.

Más recientemente se ha introducido el concepto de superficie específica, o sea, el área de la fase sólida referida a la unlidad de peso, definida por la fórmula:

$$
\mathrm{S}_{\varepsilon}=\frac{\mathrm{S}}{\mathrm{P}}\left\langle\mathrm{cm}^{z} g^{-1}\right)
$$

La industria del cemento viene utilizando, entre otros, $10 s$ aparatos denominados aTurbidimetro de Wagner» y "Permeabilimetro de Blainen para la determinación de la sitperficie específica. Se ha visto que ambos aparatos dan, para un mismo cemento, resuitados absolutamente dispares, por lo que el concepto de superficie específica, después de las determinaciones ordinarias, pierde totalmente su valor absoluto y no pasa de ser un simple indice relativo de finura, y así decimos «Stuperficie especifica Wagner» o
"Superficie especifica Blaine», según el aparato que se haya empleado para su determinación.

Se utilizan, tambien, aparatos levigadores en corriente de aire, denominados eiutriadores o flourómetros, y los residuos de levigación de cada aparato, en particular, constituyen otra forma de expresión de la finura que cabe referir a un cierto tamaño límite de partícula.

No podemos dejat de mencionat los aparatos de sedimentación en medio líquiclo, el prototipo de los cuales es la pipeta de Andreasen, y, además, otros métodos propuestos, de indudable valor científico, fundados en la adsorción de gases o de materias colorantes, todos y cada uno de los cuales presentan nuevas formas de expresión de la finura.

De esta variedad de expresiones surge una posible confusión de ideas que desde hace tiempo muchos autores van procurando aclarar.

\section{REEACIÓN ENTRE LOS RESIDUOS}

La primera cuestión que se presenta es averiguar si existe relación valedera entre los residuos de un material determinado por diversos tamices de diferente abertura de malla e, incluso, con los residuos obtenidos por levigación aérea o por sedimentación en medio líquido. 
Como es sabido, para Rosin, Rammler, Sperling, y otros, la mayor parte de los materiales pulverizados poseen una distribución de las partículas por pesos y tamaños que se ajusta con bastante aproximación a la que resulta de la fórmula exponencial:

$$
\mathrm{R}=100 e^{-\left(\frac{x}{d^{\prime}}\right)^{n}},
$$

dotide:

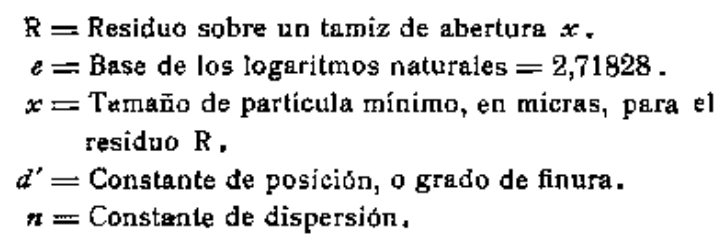

Siendo esta fórmula de difícil manejo, se acostumbra a poner bajo una forma logaritmica, con la cual es posible efectuar operaciones aritméticas.

Las transformaciones que deben efectuarse son:

$\log R=\log 100-\left(\frac{x}{d^{\prime}}\right)^{n} \log e$

$\log \left(\log 10(1-\log \mathrm{R})=n\left(\log x-\log d^{\prime}\right)+1+\log \log \epsilon\right.$.

\section{Pero:}

$e=2,71828 ; \log e=0,43429 ; \log \log \epsilon=-0,36222$.

\section{Lutego:}

$\log (2-\log \mathrm{R})=n \log x-n \log d^{\prime}-0,36222$.

Si advertimos que:

$$
n \log d^{\prime}+0,36222=\text { Constante }
$$

podemos escribir :

$$
\log (2-\log R)=n \log x-\text { Constante }
$$

De donde, el valor de la constante de dispersión :

$$
n=\frac{\Delta \log (2-\log \mathrm{R})}{\Delta \log x} .
$$

Representando la distribución granulométrica en un gráfico, donde las abscisas correspondan a los tamaños de partícula en escala logarítmica y las ordenadas a los residuos en escala doble logarítmica, la expresión [3] se convierte en la ecuación de una linea recta, ciya inclinación viene precisamente definida por lá derivada:

$$
\operatorname{tg} z=\frac{d y}{d x}=n .
$$

Según Tyudyo, en el cemento Portland:

$$
0.921<n<1,089
$$

$y$, para Anselm:

$$
0,890<n<1,110
$$

Cuanto mayor sea el ángulo $\alpha$, menor será la dispersión de las partículas. Un cemento monograinlar tendría $\alpha=90^{\circ}$ y $n=\infty$. Luego, la constante $n$ es inversa de la dispersión del material (fig. 1).

Para la aplicación de la fórmula [3] nos hace falta conocer, además de $n$, el valor de $d$.

Evidentemente que:

$$
\begin{aligned}
& \left.n \log d^{x}=n \log x-\log (2-\log \mathrm{R})-0,3622\right] \\
& \log d^{\prime}=\log x-\frac{\log (2-\log \mathrm{R})+0,36222}{n} \cdot\{[5]
\end{aligned}
$$

En cuanto al significado de la constante $d^{*}$ lo comprenderemos inmediatamente haciendo en [2] $x=d$,

$$
\begin{aligned}
\mathrm{R}^{\prime} & =100 e^{-1^{\prime \prime}} \\
\log \mathrm{R}^{\prime} & =2-\log \varepsilon=0,96571 \\
\mathrm{R}^{\prime} & =36,788
\end{aligned}
$$

La constante de posición $d^{y}$ corresponde, pues, al tamaño de partícula que tiene un residuo igual a 36,789 por 100 . 


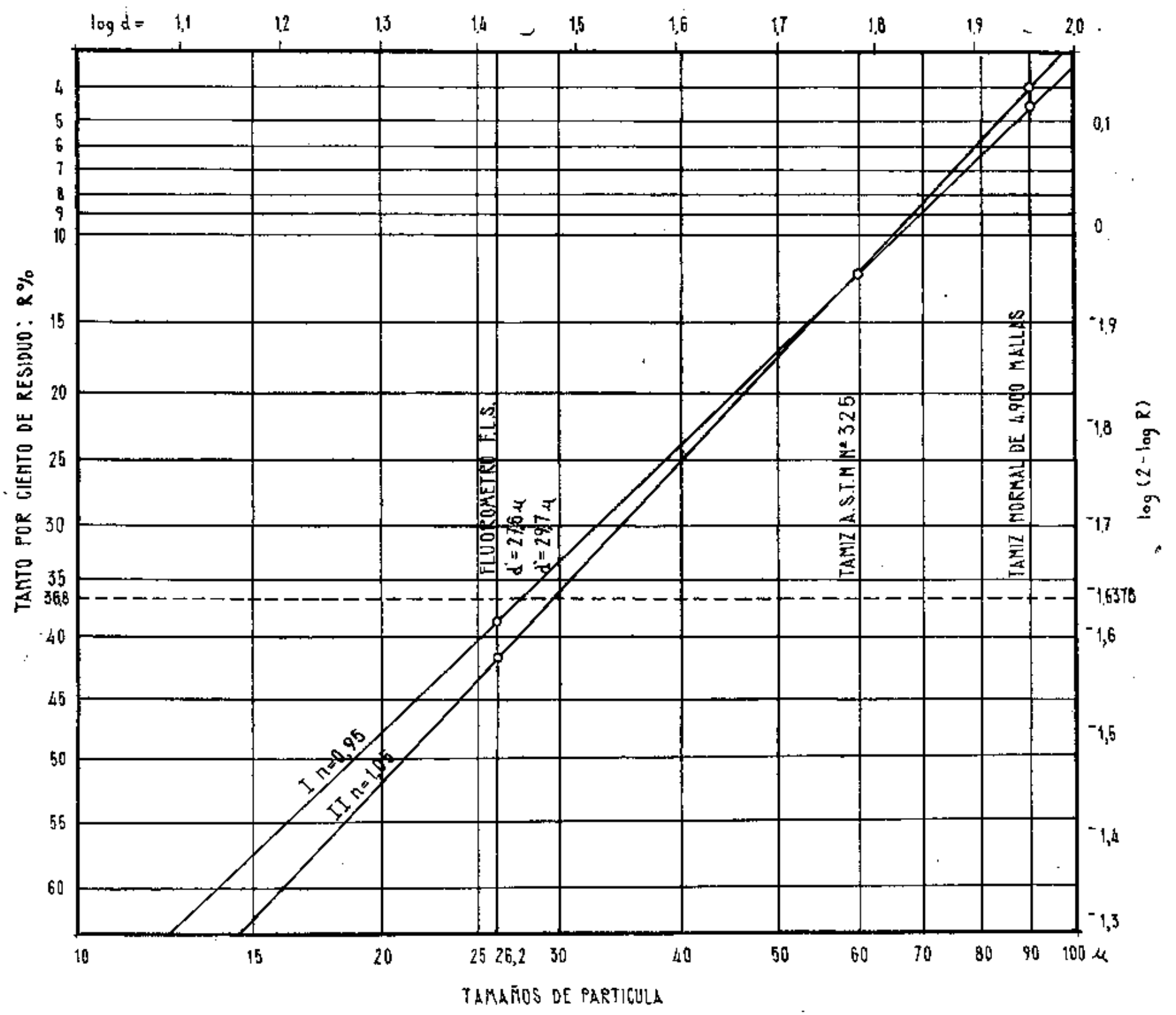

Fig. 1, 
Si derivamos dos veces la ecuación [2] vemos que la segunda derivada se anula para $\boldsymbol{x}=d^{\prime}$; esto significa que $d^{\prime}$ ' corresponde al punto de inflexión de la curva granulométrica, a bien al máximo de la curva
En la figura 2 hemos representado dos sistemas de finura equivalente por ser de un mismo orden los productos $n \times d^{\prime}$.

Después de calculados $n$ y $d^{2}$, substituyendo sus valores en la fórmula [3] podre-

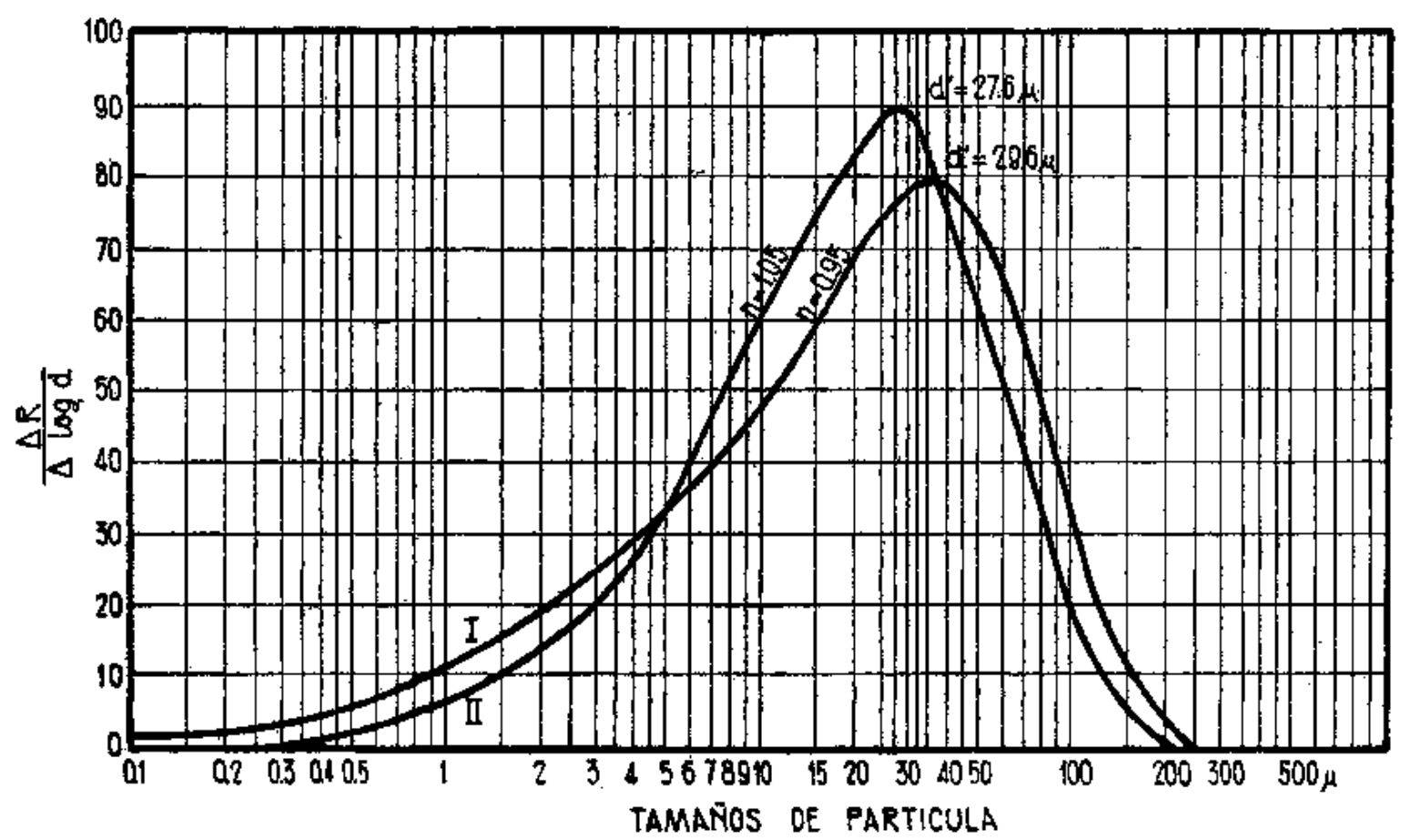

Fig. 2.

de la función derivada, lo cual se pone de manifiesto en la figura 2.

En dicha figura se dan las curvas de dos sistemas pulverulentos que siguen la fórmula exponencial [2] y que presentan diferentes valores de $n$ y $d^{\prime}$.

La curva I tiene menor dispersión o, si se quiere, mayor densidad. La curva II presenta una menor densidad y una mayor dispersión y su grado de finura aparece desviado para un tamaño de partícula mayor. Como sea que ni $n$ ni $d$ ' caracterizan por sí solos ta finura del sistema, entenderemos que ésta se caracterizará mejor por el producto de ambas magnitudes, o sea, por $n \times d$, y un material será tanto más fino cuanto menor sea el producto de sus constantes $n \times d^{\prime}$. mos calcular el valor del residuo $\mathrm{R}$ correspondiente a cualquier valor del tamaño de partícula $x$.

\section{CÁlCULO DE La SUPERFicIE ESPECfFICA}

El cálculo teórico de la supercie específica correspondiente a una sustancia pulverulenta de distribtución granulométrica conocida, es posible derivando la ecuación exponencial e integrando después entre los tamaños límites que, por ejemplo, pueden ser 100 y 0,1 micras. Este cálculo conduce a utı desarrollo en serie bastante complejo y costoso de resolver. Los xesultados que se obtienen, por otra parte, no se asemejan en 
nada a los proporcionados por el permeabilímetro o por el turbidímetro, por lo que obviaremos este punto, fácil de encontrar, por supuesto, en la bibliografía que damos al final del presente artículo.

La imprecisión del método integral reside en el desconocimiento de los límites reales de la extensión granulométrica. Wagner, al calcular la superficie específica por medio del turbidímetro, obvia estos inconvenientes admitiendo que la totalidad del cemento comprendido entre 7,5 micras y 0 micras tiene un tamañio medio de partícula igual a 3,75 micras. Tampoco es correcta la adopción de la media aritmética para expresar el tamaño medio de la fracción de peso comprendido entre dos lecturas sucesivas del turbidímetro, pero, como lo que pretendemos es referir la granulometría de un material que sigue la ley exponencial a la superficie específica calculada por el turbidimetro, aceptaremos como valederas las hipótesis de Wagner, aun sabiendo que, en ciertos puntos, son erróneas o, por lo menos, discutibles. También, por el mismo motivo, aceptaremos que el residuo por vía húmeda sobre el tamiz americano número 325 corresponde a tn tamaño tnínimo de partícula igual a 60 micras.

$\Lambda$ ceptadas estas premisas, las superficies parciales se calculan por la siguiente ecuación :

$$
\Delta \mathrm{S}=\frac{6 \times 10^{4}}{\rho} \frac{\Delta \mathrm{R}}{x_{m}}
$$

donde :

$p=$ Peso específico del material ensayado (Valor medio do $p$ para los cementos ensayados: $p=$ $\left.=3,1 \mathrm{~g} \mathrm{~cm}^{-8}\right)$.

$\Delta \mathrm{R}=$ Aumento del tanto por ciento de peso del residuo rétanido entre los tamaños de partícula $x_{1}$ y $x_{2}$.

$x_{m}=$ Tamaño medio de particula (Media aritmética entre $x_{1}$ y $\left.x_{z}\right)$,
Sustituyendo en [6] el peso específico o por su valor 3,1:

$$
\Delta \mathrm{S}=193,55 \frac{\Delta \mathrm{R}}{x_{m}} .
$$

Hemos calculado, por este procedimiento, las superficies específicas correspondientes a siete granulometrías teóricas, con los resul-

\begin{tabular}{|c|c|c|c|c|c|}
\hline \multirow{2}{*}{ Ejemplos } & \multirow{2}{*}{$n$} & \multirow{2}{*}{$d^{*}$} & \multirow{2}{*}{$n d^{\prime}$} & \multicolumn{2}{|c|}{ SUPERFICIE + SPHCIFICA } \\
\hline & & & & Integradu & Calcutasu \\
\hline 1 & 1,00 & 27,08 & 27,08 & 1.931 & 1.9332 \\
\hline 2 & 1.00 & 28,59 & $\div 8,59$ & 1.869 & $1.86 \%$ \\
\hline 3 & 1,05 & 28,07 & 29,47 & 1.838 & 1.833 \\
\hline 4 & 0,95 & 31,02 & $29,4 i$ & 1.827 & 1.833 \\
\hline 5 & 1.00 & 30,20 & 30,20 & 1.805 & 1.806 \\
\hline$b$ & 1,08 & 28,48 & $39), 76$ & 1.792 & 1.786 \\
\hline 7 & 1,00 & 40,00 & 40,00 & 1.534 & 1.538 \\
\hline Media & 1,01 & 30,49 & 30,79 & 1.795 & 1.799 \\
\hline
\end{tabular}
tados siguientes:

Ya con estos ejemplos podemos comprobar que, de conformidad con las conclusiones a que liegaron anteriormente Anselm y otros autores, la superficie específica inte. grada es inversamente proporcional al pro ducto $\mathrm{n} \times \mathrm{d}$ '. Esta relación, en su aceptación más generalizada, se puede expresar por la ecuación :

$$
\mathrm{S}_{e}=\frac{\mathrm{A}}{n d^{2}} \cdot \mathrm{B}\left(\mathrm{cm}^{2} g^{-1}\right),
$$

siendo $\mathrm{A}$ y $\mathrm{B}$ dos constantes que no dependen de la constitución granulométrica.

Como sea que interesa menos obtener el valor absoluto de la superficie especííca de un polvo, valor absoluto que, por otra parte, exigiría una discusión extensa y totalmente inadecuada a nuestro propósíto, daremos a $\mathrm{A}$ y $\mathrm{B}$ valores en concordancia con los resultados que en la práctica acostum. bramos a encontrar cuando ensayamos ce- 
mentos normales por medio del turbidímetro de Wagner. Para los ejemplos teóricos que se han expuesto en la tabla anterior, hemos obtenido:

$$
S_{e}=\frac{33.000}{n d^{\prime}}+713\left(\mathrm{~cm}^{2} g^{-1}\right) .
$$

Repitamos, sin embargo, que estos ejemplos se basaban sobre granulometrías teóricas, que seguían rigurosamente la fórmula exponencial entre los tamaños de 90 y 7,5 micras.

\section{SUPERlicie ESPECÍficA DE LOS CEMENTOS PORTLAND NORMaLES}

$\mathrm{Ya}$ expusimos en un anterior estudio nuestro, que la granulometría de los cementos Portland normales no se ajustaba perfectamente a la fórmula exponencial, principalmente para los pequeños tamaños de partícula. Como consecuencia de esta falta de ajuste, no se ptiede esperar que la aplicación de la fórmula [9] dé resultados absolutamente coincidentes con los proporcionados por la práctica.

Para determinar la superficie específica de los cementos normales, hemos seguido los conocidos métodos del turbidímetro de Wagner $\mathbf{y}$ del permeabilimetro de Blaine. La ventaja del primero reside en que nos permite determinar la curva de distribución granulométrica entre 60 y 7,5 micras. De esta curva deduciremos los valores numéricos de $n \mathrm{y}$ de $d^{\prime}$ que entran en la ecuación [8]. $\mathrm{E} 1$ permeabilímetro de Blaine, por el contrario, sólo nos proporciona un valor de conjunto, bastante más elevado que la superficie especifica calculada por el turbidímetro, pero no da ninguna indicación acerca de las constantes $n$ y $d^{\prime}$. La relación media entre ambas superficies específicas fué establecida por una simple fórmula aditiva:

$$
\mathrm{S}_{\mathrm{B} 1}=\mathrm{S}_{w}+1.890\left(\mathrm{~cm}^{\mathrm{g}} \mathrm{g}^{-1}\right)
$$

Hemos tomado al azar los resultados de 150 determinaciones de finura cubriendo una extensa gama de superficies específicas Wag ner comprendidas entre 2.700 y $1.200 \mathrm{~cm}^{2} \mathrm{~g}^{-1}$. $\mathrm{El}$ ajuste de los coeficientes $\mathrm{A}$ y $\mathrm{B}$ de la fórmula [8] se ha efectuado procurando los minimos errores. Los resultados obtenidos, convenientemente redondeados, nos condujeron a la fórmula :

$$
\mathrm{S}_{w}=\frac{65.000}{n \times d^{2}}-400\left(\mathrm{~cm}^{2} g^{-1}\right)
$$

Combinando $[10]$ y. [11]:

$$
\mathrm{S}_{\mathrm{B1}}=\frac{65.000}{n \times d^{2}}+1.490\left(\mathrm{~cm}^{2} g^{-1}\right) .
$$

Comparando las fórmulas [9] y [11] se observa un punto de coincidenciá, que viene determinado por:

$$
\begin{aligned}
\frac{65.000}{n \times}-400 & =\frac{33,000}{n \times d^{\prime}}+713 \\
n \times d^{\prime} & =28,75 \\
\mathrm{~S}_{w} & =1.861 \mathrm{cnl}^{2} g^{-1}
\end{aligned}
$$

Para superficies cspecificas superiores a $1.861 \mathrm{~cm}^{2} \mathrm{~g}^{-1}$, obtenemos, con la fórmula [11], resultados mayores que los calculados por medio de la fórmula [9]. Si la superficie específica es menor que $1.861 \mathrm{~cm}^{2} \mathrm{~g}^{-1}$, los resultados calculados por aplicación de la fórmula [11] son menores que cuando son obtenidos por la fórmula [9].

Sin embargo, tanto una fórmula como otra, y en general, todas las fórmulas análogas a la ectuación [8], presentan el inconveniente de exigir el conocimiento de $n$ y $d^{\prime}$, constantes que no se pueden obtener por medio de determinaciones directas, sino que obligan a cálculos que exigen un cierto tiempo para su ejecución.

El problema de la correlación de los residuos con las superficies específicas sólo ad- 
quiere interés si conseguimos proponer un método que nos conduzca a la soltución fácilmente, con una simplicidad que haga innecesarios todos Ios cálculos.

Esta solución ideal solamente puede proporcionarla un ábaco de puntos alineados o nomograma (figura 3).

\section{Trazado del nomograma: escalas di LOS RESIDUOS}

El nomograma que vamos a presentar expresa los residuos en escalas logarítmicas sobre lineas verticales y paralelas, $x_{1}, x_{3}, x_{3}$, correspondiendo cada línea a un tamaño de partícula determinado. Hemos escogido los tamaños de partícula siguientes:

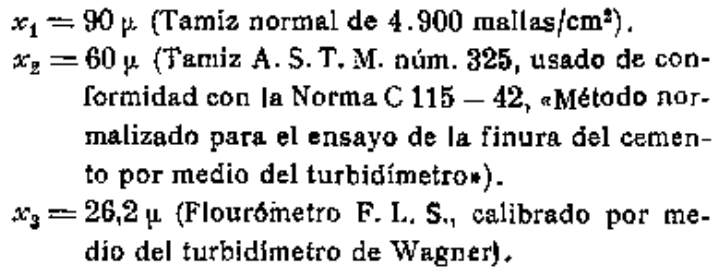
formidad con la Norma C $115-42$, aMétodo normalizado para el ensayo de la finura del cemento por medio del turbidímetron).

$x_{3}=26,2 \mu$ (Flourómetro F. L. S., calibrado por medio del turbidímetro de Wagnery.

En el nomograma se pretende que, cuando la constante granulométrica de dispersión $n$ sea igual a la unidad, los residuos $\mathrm{R}_{1}, \mathrm{R}_{2}$ y $R_{3}$ queden alineados sobre una recta horizontal, en los puntos donde corta a las verticales $x_{1}, x_{2}$ y $x_{3}$. Para ello es necesario que las escalas logarítmicas correspondientes a cada tamaño de partícula guarden entre sí una relación inversa a los dichos tamaños, ya que, para $n=1$, la fórmula [2] se reduce a :

$$
\begin{array}{r}
\mathrm{R}=100 e^{-\frac{x}{d^{\prime}}} \\
\frac{\mathrm{R}}{100}=r=e^{-\frac{x}{d^{5}}} .
\end{array}
$$

Pasando a los logaritmos neperianos:

$$
\left.\begin{array}{l}
\log r=-\frac{x}{d^{\prime}} \\
\frac{\log r}{x}=-\frac{1}{d^{\prime}}=\text { Constante } .
\end{array}\right\}
$$

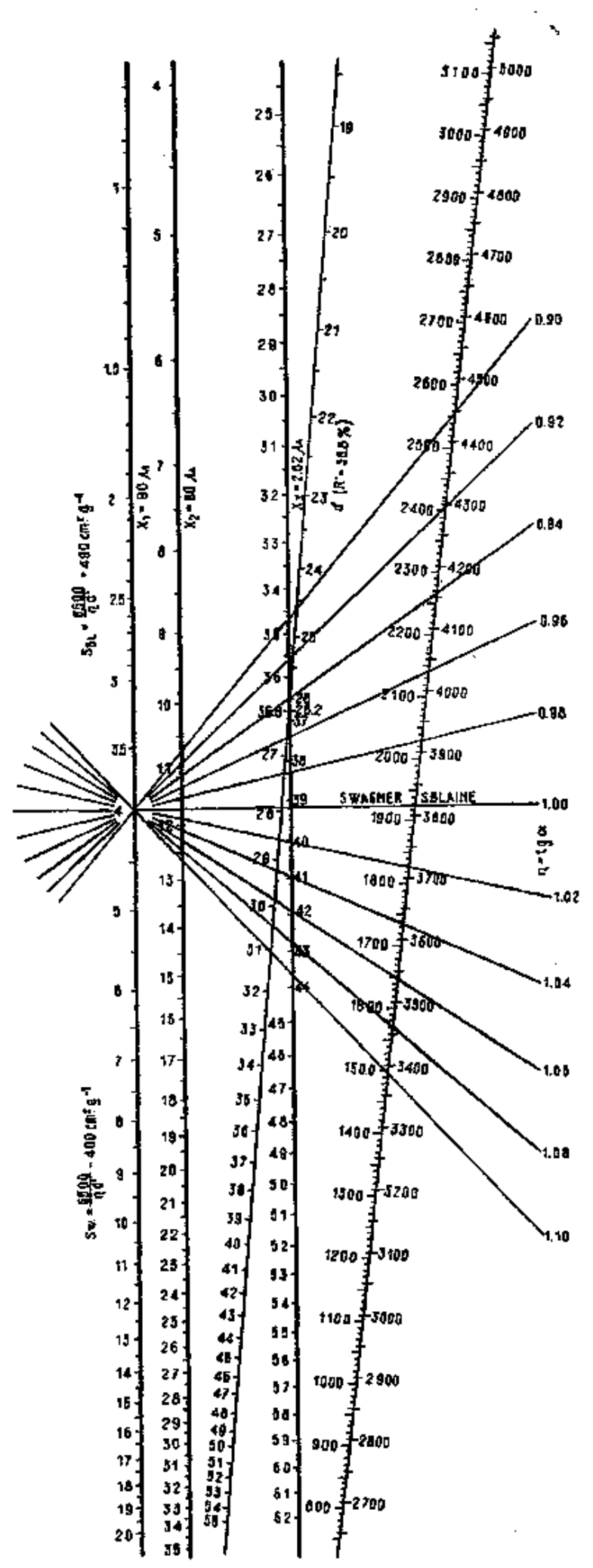

Fig. 3. 
Cuando $n \neq 1$, para que los puntos representativos de los residuos continúen con alineación recta es necesario que la separación entre las líneas paralelas verticales sea proporcional a la diferencia de logaritmos de los tamaños de partícula $\Delta \log x$, de acuerdo con el denominador de la expresión [4]. Así, para los tamaños de partícula que hemos escogido, tendremos:

\section{E S C A I. A S}

\begin{tabular}{|c|c|c|c|}
\hline & 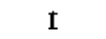 & II & 111 \\
\hline Tamaños de particula....... & $90 \mathrm{~g}$ & $60 \mu$ & 86,214 \\
\hline Relación de escalas......... & 1,000 & $1,5 \notin 0$ & 3,162 \\
\hline $\log x \ldots \ldots \ldots+\ldots \ldots \ldots$ & 1,95424 & 1,77815 & 1,41930 \\
\hline$\Delta \log x \ldots \ldots \ldots \ldots \ldots \ldots \ldots$ & 0,00000 & 0,17809 & 0,58594 \\
\hline Intervalo, on men. ${ }^{\prime}, \ldots \ldots \ldots$ & 0,00 & 80,44 & 100,00 \\
\hline \multicolumn{4}{|l|}{ Algunos residuos básitos $(\mathrm{n}=1)$} \\
\hline$d^{\prime}=27,98 \mu \ldots \ldots \ldots \ldots \ldots$ & 4,00 & 11,70 & 39,18 \\
\hline$d^{\prime}=30 \quad \mu, \ldots \ldots \ldots$, & 4,97 & 18,53 & 41,76 \\
\hline$d^{\prime}=85,63 \mu \ldots \ldots \ldots \ldots \ldots+$. & 8,00 & 18,57 & 47,94 \\
\hline$d^{\prime}=40 \quad[n \ldots \ldots \ldots \ldots \ldots$ & 10,54 & 22,91 & 51,04 \\
\hline
\end{tabular}

Como sea que el nomograma se aproxima suficientemente a los resultados del cálculo numérico, creemos innecesario dar más ejemplos de la correlación de los residuos y remitimos al lector a la observación del nomograma para todos los casos de correspondencia.

Cualesquiera otros tamices o flourómetros, con separación de partículas en tamaños no representados en nuestro nomograma, pueden ser muy fácilmente introducidos trazando las correspondientes rectas paralelas, sujetando las escalas logarítmicas de residuos y los intervalos de separación a las Normas anteriormente expuestas.

\section{REPRESENTACKÓN DE $n$ Y $d^{\prime}$ EN EL NOMOGRAMA}

En nuestro nomograma la constante de dispersión $n$ se representa por la inclinación de la recta granulométrica. Por construcción, cuando $n=1$ la recta es horizontal.

Cuando $n<1$, la recta aparece con inclinación positiva, aumentando la pendiente con la diferencia $n-1$. Con la pendiente aumenta la dispersión de los tamaños de partícula y la superficie específica del cemento.

Cuando $n>1$, la recta granulométrica presenta inclinación negativa, que aumenta con el valor de $n$. Los valores crecientes de $n$ indican una mayor concentración de tamaños de partícula y una menor superficie específica.

En el nomograma aparecen representadas 11 granulometrías, con un punto común: el residuo sobre el tamiz de 4.900 mallas $/ \mathrm{cm}^{2}$, que para todos los once casos se supone igual a 4 por 100. La constante $n$ varía de 0,9 a 1,1 , y de esta variación resulta:

\begin{tabular}{c|c|c|c}
\hline$v$ & $R_{1}$ & $R_{2}$ & $R_{3}$ \\
\hline & & & \\
0,90 & 4,0 & 10,70 & 34,64 \\
0,92 & 4,0 & 10,90 & 35,05 \\
0,94 & 4,0 & 11,10 & 36,46 \\
0,96 & 4,0 & 12,30 & 37,37 \\
0,98 & 4,0 & 11,50 & 38,27 \\
1,00 & 4,0 & 11,70 & 39,18 \\
1,02 & 4,0 & 12,11 & 40,98 \\
1,04 & 4,0 & 12,11 & 40,98 \\
1,06 & 4,0 & 12,32 & 41,89 \\
1,08 & 4,0 & 12,52 & 42,79 \\
1,10 & 4,0 & 12,74 & 43,69 \\
\hline
\end{tabular}

La constante de posición, o grado de finura $d$, es decir, el tamaño de partícula correspondiente a un residuo $R^{\prime}=36,8$ por 100 , se puede obtener sobre el propio nomograma. Por la fórmula [13] se advierte que los valores del grado de finura $d$ ' se deben representar sobre una línea recta, graduada con una escala de números recíprocos.

La recta sobre la cual leeremos los valores de $d$ ' no será una paralela a las escalas lo- 
garitmicas de los residuos, sino una línea inclinada, la cual, para $d^{\prime}=26,2 \mu$, cortará a los residuos del tamaño $x_{3}$ sobre el porcentaje de 36,8 , lo que nos dice: cuando el flou rómetro F. L. S., con separación de partículas a $26,2 \mu$ proporcionan un residuo de 36,8 por 190 , el grado de finura del cemento ensayado será precisamente $d^{\prime}=26,2 \cdot \mu$. Para residuos $\mathrm{R}_{3}>36,8$, $d$ ' queda interpolado entre $x_{2}$ y $x_{3}$. Para residuos $\mathrm{R}_{3}<36,8$, $d$ ' se extrapola para tamaños inferiores a $x_{3}$.

Para el trazado de la recta $d$ ' en el nomograma nos hemos servido de los puntos siguientes:

\begin{tabular}{c|r|r|r|r}
\hline$n$ & \multicolumn{1}{|c|}{$R_{1}$} & \multicolumn{1}{|c|}{$R_{2}$} & $R_{3}$ & $d^{\prime \prime}$ \\
\hline 1,00 & 1,11 & 4.97 & 26,98 & 20 \\
0,63 & 7,55 & 13,53 & 30,55 & 20 \\
1,00 & 4,97 & 13,53 & 41,76 & 30 \\
1,71 & 1,83 & 13,53 & 61,56 & 40 \\
1,00 & 10,54 & 22,31 & 51,94 & 40 \\
\hline
\end{tabular}

\section{REPRESENTACIÚN DE LA SUTERTICIE ESPECÍFICA}

Jas superficies especificas que se determinan con nuestro nomograma corresponden a las fórmulas [11] y [12]. Se representan sobre tuna recta inclinada a escala natural. A la izquierda de la recta aparece la escala de superficies especificas Wagner y a la derechat la escala de superficies específicas Blaine. Habiendo adoptado para estas últimas una simple formuli de adición, todos los cálculos que nos han conducido al trazado del nomograma los hemos efectuado én función de la superficie específica Wagner, que designamos por $\mathrm{S}_{\mathrm{w}}$. La posición de la escala de stuperficies específicas en el nomograma se ba fijado con el auxilio de los puntos siguticntes :

\begin{tabular}{c|c|c|c|c|c}
\hline$n$ & ${ }^{\prime}$ & $R_{2}$ & $R_{2}$ & $R_{3}$ & $s_{w}$ \\
\hline 1,000 & 27,083 & 3,60 & 10,91 & 38,01 & 2.0100 \\
0,951 & 28,479 & 5,05 & 13,12 & 39,70 & 2.000 \\
1,000 & 29,546 & 4,75 & 13,12 & 41,20 & 1.800 \\
1,058 & 30,718 & 4,43 & 13,12 & 42,96 & 1.600 \\
1,000 & 32,500 & 6,27 & 15,78 & 44,66 & 1.600 \\
\hline
\end{tabular}

\section{\. APITCACIÓN E INTERPRETACión DEL NOMOGRAMA}

El nomograma presentado tiene especial aplicación para determinar la superficie especifica aproximada de un cemento conociendo dos de sus residuos. Al mismo tiempo se lee en el nomograma el grado de finura $d^{\prime} \mathrm{y}$. por la inclinación de la recta, se puede esti. mar el valor de la constante de dispersión $n$.

De la simple inspección de las escalas se deduce que un error de 1 por 100 en el residuo sobre el tamiz de $\mathbf{4 . 9 0 0}$ mallas puede dar lugar a considerables variaciones de $n \mathrm{y}$, por consectencia, de la superficie específica.

En general, la determinación del residuo sobre el tamiz de $\mathbf{4 . 9 0 0}$ mallas da lugar a errores groseros que to hacen totalmente inadecuado para la determinación de la sto perficie específica. Es muy preferible utilizar para la determinación de la superficie específica y del grado de finura tan solamente los residuos proporcionados por el t a miz A. S. T. M. núm. 325 y por el flourómetro. En este caso, el nomogram puede servir para detectar errores en la determinación de los residuos sobre el tamiz de $4.900 \mathrm{ma}-$ 1 las $/ \mathrm{cm}^{2}$, siempre que éste presente un valor que lo aleje notablemente de la línea recta que une los otros residuos.

E1 nomograma nos indica claramente cómo, para materiales con igual residuo sobre el tamiz de $4.900 \mathrm{malias} / \mathrm{cm}^{2}$, podemos tener muy diversos grados de finura y muy variadas superficies específicas. Todo depen- 
de de la constante de dispersión n. Así, para un residuo común de 4 por 100 sobre el antedicho tamiz, para $n=0,9$ la superficie especifica Wagner es del orden de $2.500 \mathrm{~cm}^{2} / \mathrm{g}$. $n=1,1, S_{\mathrm{w}}=1.500 \mathrm{~cm}^{2} / \mathrm{g}$. En ofras palabras, la fintura de un cemento queda absolutamente indeterminada si tan solamente conocemos el residuo que no atraviesa una red de abertura de malla conocida.

Cada molino de cemento tiene su fisonomía que se refleja en la granulometría del cemento obtenido. Los molinos con separación de aire presentan bajas dispersiones de los tamaños de partícula, o sea, la constante de dispersión $n$ es mayor que la unidad. Los cementos obtenidos serán de baja superficie específica a no ser que se obtengan muy bajos residuos sobre el tamiz de $4.900 \mathrm{ma}$ llas. En los molinos compuestos, sin separa. ción de aire y con cámaras con cuerpos mo. ledores de tamaño escalonado, se obtienen constantes de dispersión más próximas a la unidad $y$, por lo tanto, para los mismos residuos que en un molino con separación de aire se tienen mayores superficies específicas.

El nomograma, todavía, esclarece aparentes anomalías de la grantlometría de los cementos. Así, por ejemplo, dos cementos con igual residuo flourométrico pueden tener diferente superficie específica $y$, contrat lo que a simple vista parece que debiera ser, ta mayor superficie específica corresponde precisamente al cemento que tiene mayor re siduo sobre el tamiz de 4.900 mallas $/ \mathrm{cm}^{2}$. En efecto, la simple inspección del nomograma nos dice que, al aumentar $R_{1}$, permaneciendo $R_{3}$ constante, ticnden a disminuir las constantes granulométricas $n$ y $d, y$ al disminuir su producto, aumenta la superficie específica.

Las escalas de superficies específicas son convencionales. Ya dijimos que no correspondían a los valores que se obtienen según lá ecuación teóricạ [9]. Las ecuaciones [11] y [12] de que nos hemos servido presentan constantes arbitrarias, ajustadas con bastante aproximación a los valores medios que hemos obtenido en nuestro laboratorio. Oiros laboratorios, manejando diferentes tipos y calidades de cemento, puede que observen errores sistemáticos en la escala de superfi. cies específicas que, en el caso de tener interés en la aplicación de este fácil nomograma, les obliguen a reajustes de escalas. No hay inconveniente en ello, ya que, para nosotros, lo fundamental del nomograma, es la facilidad con que podemos relacionar residuos y supetficies específicas sin necesidad de cálculos logarítmicos ni de simples operaciones numéricas. Los porcentajes en peso de los residuos determinados por los procedimientos más vulgares son los datos que entran directamente en el mecanismo de este nomograma.

\section{Bibliografóa referente a La deTerminación de la finura de los cementos}

(1) ANSELM (W.): Zerkleinerungstechnik und Stawb. (l'écnica de la trituración y del polvo). «Deutscher Ingenieur Verlag G. m. b. H.», Düsseldorf, 1900, 59 págs., 29 figs., 10 tabl., 75 ref. bibl., 1 apéndice.

(2) Estudio economico comparativo de las resistencias de los cementos en relación con la molienda de los mismos. «Ultimos avances». Madrid, núm. 37, págs. 1-13, 2 figs., 1 tabl., 7 ref. bibliog.

(3) Biblograpie concernont la mesture de la finesse des matières pulverulentes. (Bibliografía relativa a la medición de la finura de los materiales pulverulentos.) aRevue des Matériauxn. Farís, nov. 1964, núm. 470, págs. 329-28, 104 referencias bibliográficas de trabajas publicados entre 1028 y 1950.

(4) BLAINE (R. J.): A simplified air permeability fineness opparatus. (Aparato simplificado para determinación de la finura por la medida de la permeabilidad al aire). Boletín A. S. T. M.*, 1916, Race St. Philadelphia 8, Pa.

(5) BoRner (H.): Broyoge du ciment en circuit owvert et en circuit fermé. (Molienda del cemento en circuito

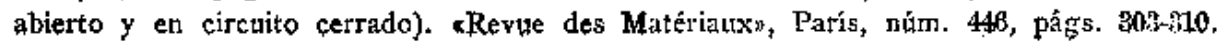


(6) BRochad (J.): Mesure de la granulonétrie et de la surface spécifique des timents. C. E. R. I. L. H., Paris. «Pub. téc. n. $20 \mathrm{~m}$, ellero 1950 , 28 págs., 26 figs., 30 ref. bibliog.

(7) GEssne (H.): L’analyse mécanique (E! análisis mecánico). «Dunod», Paris, 1936, 270 págs., 106 figs, 23 tablas.

(8) Guye (F.): Nuevos métodos para la detemminación de la finura de los cementos. aCementow, Barcelona, numeros 163 y 164, octubre $y$ noviembre 1947, págs. 306 y 322, 3 figs., 7 ref. bibliog.

(9) JASPERS (M. J. M.): Les lois régissant les liaisons entre la surface spécifique et les propriétés téchnologiques du cimsut (Las leyes que rigen las relaciones entre la superficie especifica y las propiedades tecnológicas del cemento). «Revise des Matériaux», Paris, 1951, junio a septiembte, núms. 429, 490 y 431-32, páginas 169-74, 207-15, 254-7, to tablas. 23 diagramas, 9 ref. bibliog.

(10) Inopitalier (F.) y Момот (CH.); Détermination de la granulométrie des ciments (Determinación de la granulametría de los cementos). .C. E. R. I L. H.s, París, julio 1948, publ. técnica núm. 8.

(11) Margarit (A.): Determinación de la granulometría de los comentos mediante la pipeta de Andreason modificada, aevista de Ciencia aplicadan, Madric, ním. 33, año VII, 4, julio-agosto 1953, pags. 315-20.

(12) Matouschex (F.): La granshlométrie des ciments. (La granulometria de los cementos). «Revue des Matériaux», Paris, mayo y junio de $18 \bar{x} 0$, núms. $410 \mathrm{y}$ y $41 \mathrm{~T}$, págs. 163-9, 197-201, 12 figs. 9 tablas.

(13) Rosix (P.), Rammler (E.) y SrerLing (K.): Berichtsfolge des Reichskohlentates $C 52$ (Memoria $C 52$ del Comité Técnico Económico del Carbón de Ajemania). Berlin, 1833.

(14) Steinherz (A. R.): La finesse de mouture du cimené portland artificiel (La fintra de molienda del cemento portlancl). xRevue des Matériatux, París, narzo y abríl de 1952, ním. 498-4, págs. 7ō-80 y 102-4.

(15) VILRI SEvieR1 (F.): Observaciones relativas a la dcterminación de la composición grantulométrica de los cementss. aCementos, Barcelona, febrero y marzo de 1936, núms, 81 y 82 , pàgs. 3is y 67 .

(16) Virlida (A.): Residuos y superficie especifica. Cementox, Barcelona, marzo 1051, núm, 204, págs. 71-6, 4 tablas, 1 fig.

(17) Virelua (A.): Determinación de la supericie especifica por la medita di la permeabilidad. "Cementon, Barcelona, rims. 296, 7 y 8 , noviembre y diciembse de 1958 у enero de 1954,3 tablas, 3 figs., 10 referencias bibliog.

(18) Virella (A.): Anäisis gransiométrico del cenento por el empleo conjunto del turbidinetro de Wagner $y$ del flourómetro F. L. S. calibrado a $\$ 0 \mu$. «Cemento», Barcelona, marzo, abril y mayo de 1955, nú. meros 252,3 y 1 , 12 figs., 18 ref. bibliog.

(19) Virelia (A.); Los ensayos de calidad in los laboratorios de fábricas. eSegundos coloquios de directores y técnicos de fábricas de cementos, Madrid, marzo 1957.

(20) WaGner (L. A.): A rapid method for the deternination of the specific surface of portlond cement. (Método rápido para la determinación de la superficie especifica del cemento portland). «Proceclings A. S. T. M.s, Fhiladelphia, 1953, vol. 83, II, págs. 5̋3-70. Versión española publicada en cementos, Batcelona, octubre : noviembre 1934, nums. 65-6.

(21) WhLTHER (H.): Feinheitanalyse und speziftsche Oberfläche von Mineralpulvem. (Análisis de la finura y superficie específica de los polvos minerales). Silikattechnik, dicıembre 1963, núm. 12, 21 figs., 6 tablas, 29 ref. bibliog.

(22) WeIDENHAMMER (F.): Berechnung del Oberfläche eines kornigen Gutes rach Rosin Rammler. (Determinación de la superficie de un material granulado, seguin la ley de Rosin y Rammler). CTonitsdustrien, núms. 9 y 10 (mayo 1961), págs. 133-5, 3 figs., 5 ref. bibliog.

\section{NOTA SOBRE LAS REFERENCIAS BIBLIOGR.́fICAS}

Para una bibliografía mucho más completa sobre li. finura y la superficie especifíca de los cementos remitimos al lector a nuestra llamada nún. (3). Nuestras referencias bibliográficas se limitan a aquellos trabajos que nos han servido para la preparación de nuestro estudio, așí como a nuestros trabajos anteriormente publicados sobre el mișmo tema. 